\title{
Potential microRNA biomarkers for acute ischemic stroke
}

\author{
YE ZENG $^{1}$, JING-XIA LIU ${ }^{1}$, ZHI-PING YAN ${ }^{1}$, XING-HONG YAO $^{2}$ and XIAO-HENG LIU ${ }^{1}$ \\ ${ }^{1}$ Institute of Biomedical Engineering, School of Preclinical and Forensic Medicine, Sichuan University, Chengdu, \\ Sichuan 610041; ${ }^{2}$ State Key Laboratory of Oncology in South China, Department of Radiation Oncology, \\ Sun Yat-Sen University Cancer Center, Guangzhou, Guangdong 510060, P.R. China
}

Received March 11,2015; Accepted September 29, 2015

DOI: 10.3892/ijmm.2015.2367

\begin{abstract}
Acute ischemic stroke is a significant cause of high morbidity and mortality in the aging population globally. However, current therapeutic strategies for acute ischemic stroke are limited. Atherosclerotic plaque is considered an independent risk factor for acute ischemic stroke. To identify biomarkers for carotid atheromatous plaque, bioinformatics analysis of the gene microarray data of plaque and intact tissue from individuals was performed. Differentially expressed genes (DEGs) were identified using the Multtest and Limma packages of $R$ language, including 56 downregulated and 69 upregulated DEGs. Enriched microRNA (miRNA or miR) DEGs networks were generated using WebGestalt software and the STRING databases, and the miRNAs were validated using serum from acute ischemic stroke patients with reverse transcription quantitative PCR (RT-qPCR). Four confirmed differentially expressed miRNAs (miR-9, -22, -23 and -125) were associated with 28 upregulated DEGs, and 7 miRNAs (miR-9, -30, -33, -124, -181, -218 and -330) were associated with 25 downregulated DEGs. Gene ontology (GO) function suggested that the confirmed miRNA-targeted DEGs predominately associated with signal transduction, the circulatory system, biological adhesion, striated muscle contraction, wound healing and the immune system. The confirmed miRNA-targeted genes identified serve as potential therapeutic targets for acute ischemic stroke.
\end{abstract}

\section{Introduction}

Stroke is one of the most common causes of mortality and disability worldwide (1-3). Stroke is a clinical syndrome with pathological types including ischemic, intracerebral hemorrhage, and sub-arachnoid hemorrhage types $(4,5)$. Stroke

Correspondence to: Dr Ye Zeng or Dr Xiao-Heng Liu, Institute of Biomedical Engineering, School of Preclinical and Forensic Medicine, Sichuan University, No. 17, Section 3, Renmin South Road, Chengdu, Sichuan 610041, P.R. China

E-mail: ye@scu.edu.cn

E-mail: liuxiaohg@scu.edu.cn

Key words: stroke, acute, atherosclerotic plaque, microRNA, biomarkers risk increases with age (6). Ischemic stroke, which accounts for $87 \%$ of all stroke subtypes presented clinically (7), is prevalent in populations of European origin and Chinese populations $(5,8,9)$. Acute ischemic stroke is the major cause of high morbidity and mortality in the aging population worldwide (10). The prognosis is worse for stroke, with up to $50 \%$ of the individuals worldwide confirmed to have suffered a stroke succumbing or being dependent on carers 6 months after the event (5). Current therapeutic strategies for acute ischemic stroke are limited (3). Intravenous thrombolysis with aspirin improves the outcome for only a small proportion of patients presenting early after symptom onset with acute ischemic stroke (11). Thus, seeking clinical diagnosis, prognostic biomarkers and therapeutic targets for ischemic stroke is indispensable. Approximately $50 \%$ of ischemic strokes are attributed to large-artery atherothrombotic disease, $25 \%$ to disease of the small intracranial arteries, $20 \%$ to cardiac emboli, and 5\% to various rare causes (e.g., extracranial artery dissection) $(4,5)$. The most common characteristic of acute ischemic stroke is an occlusion of the artery. Plaque composition is thought to be an independent risk factor for acute ischemic stroke (12).

The thickness of the fibrous cap, the size of the necrotic core and intraplaque hemorrhage, and the extent of inflammatory activity within the plaque are key factors in advanced plaque that are most likely to lead to complications (13). However, it was not possible to demonstrate how the complicated plaques associate with symptoms, since it appears that complicated plaques may occur at any time, irrespective of symptoms (14). Additionally, the exact mechanisms causing plaque rupture remain to be determined (15). Detection of vulnerable plaque is important for preventing future clinical events and remains to be resolved.

MicroRNAs (miRNAs or miRs) are non-coding RNAs that regulate gene expression by translation inhibition or messenger RNA degradation (16). miRNAs that are highly stable in serum (17) and urine (18) are potential biomarkers of pathology, such as stroke $(19,20)$ and age-associated pathologies (21). The differential expression of miRNAs in peripheral blood of young ischemic stroke patients in Malaysia has been previously examined (22). However, the miRNAs involved in the formation and progression of atherosclerotic plaque have yet to be thoroughly investigated.

An intra-patient comparison on the transcript profile of atheromatous plaque and intact tissue samples was performed by Ayari and Bricca (23). However, those authors only analyzed 
Table I. Top 10 enriched miRNA with target DEGs.

\begin{tabular}{|c|c|c|c|}
\hline Up-/Downregulated & Enriched miRNAs & Targets DEGs & Adj.P \\
\hline \multirow[t]{10}{*}{ Downregulated } & hsa_TGTTTAC, miR-30 & $\begin{array}{l}\text { LGI1|ACTC1|PRUNE2|NLGN1|GRIA2|FRK| } \\
\text { TTLL7|LPHN3|PCDH20 }\end{array}$ & 0.0006 \\
\hline & hsa_GTGGTGA, miR-197 & NLGN1|GRIA2|TTLL7|LPHN3 & 0.0006 \\
\hline & hsa_AAGCCAT, miR-135 & THRBILRRN1|FRKIPRUNE2|PDE8B|NEGR1 & 0.0029 \\
\hline & hsa_TGCCTTA, miR-124 & RYR2|THRB|CNTN3|GRIA2|CNTN1|NEGR1 & 0.0261 \\
\hline & hsa_TGAATGT, miR-181 & THRB|LRRN1|CNTN4|GRIA2|NEGR1 & 0.0563 \\
\hline & hsa_TGCTTTG, miR-330 & PDZRN3|PCDH11Y|FRKIPRUNE2 & 0.0609 \\
\hline & hsa_CAATGCA, miR-33 & LGI1ICNTN4 & 0.0864 \\
\hline & hsa_TAGCTTT, miR-9 & THRBICNTN4INEGR1 & 0.0864 \\
\hline & hsa_AAGCACA, miR-218 & PLD5|GRIA2|FREM1INPY1R & 0.0864 \\
\hline & hsa_TGCAAAC, miR-452 & PLCB4ITHRB & 0.0915 \\
\hline \multirow[t]{6}{*}{ Upregulated } & hsa_AATGTGA, miR-23 & PRDM1ICD163 & 0.1423 \\
\hline & hsa_ACCAAAG, miR-9 & VAV3IPRDM1 & 0.1423 \\
\hline & hsa_GGCAGCT, miR-22 & DPP4IAQP9 & 0.1423 \\
\hline & hsa_ACTGTGA, miR-27 & VAV3IRGS1 & 0.1423 \\
\hline & hsa_CTTTGTA, miR-524 & VAV3IPRDM1 & 0.1423 \\
\hline & hsa_CTCAGGG, miR-125 & PRDM1IANPEP & 0.1423 \\
\hline
\end{tabular}

Top 10, 10 most significant categories; Adj.P, P-value from hypergeometric test adjusted by multiple test adjustment (Benjamini-Hochberg method); DEGs, differentially expressed genes; miRNA or miR, microRNA.

the expression of CD163 and heme oxygenase (decycling) 1 (HMOX1), which are involved in the homeostasis of iron and heme in atherosclerotic plaques (23).

In the present study, we aimed to identify which miRNAs were associated with the formation and progression of plaque, to investigate the potential mechanism of plaque vulnerability to rupture, and to examine potential biomarkers and therapeutic targets for acute ischemic stroke. The same microarray data as that used by Ayari and Bricca (23) were downloaded, and differentially expressed genes (DEGs) in paired atheromatous plaque and intact tissue samples were identified using the Multtest and Limma packages of $\mathrm{R}$ language. Enriched miRNAs that regulate the DEGs were then predicted using WebGestalt software. The expression of those predicted miRNAs in serum was confirmed using reverse transcription quantitative PCR (RT-qPCR). Protein-protein interactions (PPIs) of DEGs were constructed from STRING databases (http://string-db.org/), and confirmed miRNA-targeted DEGs interactions were visualized using Cytoscape software (http://www.cytoscape.org/). Gene ontology (GO) function and pathway analysis of confirmed miRNA-targeted DEGs were performed using the Bingo plug-in of Cytoscape software.

\section{Materials and methods}

Microarray data and data preprocessing. The gene microarray data GSE43292 (23) was downloaded from the Gene Expression Omnibus (GEO), which includes paired atheromatous plaque and intact tissue samples collected from 32 patients. Endarterectomy specimens were characterized histologically according to the classification proposed by the Nomenclature Committee of the American Heart Association (24). The sections consisted of the intima and a majority of tunica media, although the tunica adventitia was excluded. Stage IV and later were considered as atheromatous plaques, and intact tissues were almost exclusively composed of stage I and II lesions (23). The platform of GSE43292 was GPL6244 (HuGene-1_0-st) Affymetrix Human Gene 1.0 ST Array [transcript (gene) version].

The mean expression of a total of 33,297 probes was derived from GEO, and accurate annotations were made. Probes matching $>1$ gene ID were discarded. All the probes were then mapped to Entrez gene IDs.

Screening of the DEGs. The $\log 2$ transformation of the expression value was performed for each probe (25). Up- and downregulated probes were determined according to their fold differences (26). DEGs were identified using the Multtest and Limma packages of R language (27). The false discovery rate (FDR) correction was calculated using the Benjamini- Hochberg (BH) method (FDR < 0.05) (28). A two-fold change method was used to identify DEGs (Ilog FC (Fold change) $>1$ ).

Prediction of enriched miRNAs in carotid atheroma. The WebGestal, a web-based gene set analysis toolkit, was used to predict miRNA associated with DEGs $(29,30)$. The hypergeometric test was widely used to investigate the significance of functional terms enrichment within a list of genes. The 10 most significant categories (Top 10) and a minimum of two genes for a category were selected as the threshold. 
Table II. DEGs in the confirmed miRNA-target DEGs interaction networks.

\begin{tabular}{|c|c|c|c|c|}
\hline Gene symbol & Entrez ID & $\operatorname{Adj} . \mathrm{P}$ & $\log \mathrm{FC}$ & Gene name \\
\hline CNTN1 & 1272 & $2.00 \mathrm{E}-05$ & -1.91103 & Contactin 1 \\
\hline CNTN4 & 152330 & $1.70 \mathrm{E}-05$ & -1.79233 & Contactin 4 \\
\hline CASQ2 & 845 & $1.91 \mathrm{E}-05$ & -1.66766 & Calsequestrin 2 (cardiac muscle) \\
\hline PCDH11Y & 83259 & $2.55 \mathrm{E}-05$ & -1.38541 & Protocadherin 11 Y-linked \\
\hline CNTN3 & 5067 & $3.89 \mathrm{E}-05$ & -1.36174 & Contactin 3 (plasmacytoma associated) \\
\hline NPY1R & 4886 & $8.70 \mathrm{E}-05$ & -1.27438 & Neuropeptide Y receptor Y1 \\
\hline LRRN1 & 57633 & $2.85 \mathrm{E}-05$ & -1.27233 & Leucine rich repeat neuronal 1 \\
\hline RYR2 & 6262 & $5.41 \mathrm{E}-05$ & -1.24144 & Ryanodine receptor 2 (cardiac) \\
\hline LGI1 & 9211 & $6.74 \mathrm{E}-05$ & -1.15644 & Leucine-rich, glioma inactivated 1 \\
\hline PRUNE2 & 158471 & $3.53 \mathrm{E}-05$ & -1.11997 & Prune homolog 2 (Drosophila) \\
\hline PCDH20 & 64881 & $2.81 \mathrm{E}-05$ & -1.11425 & Protocadherin 20 \\
\hline PLD5 & 200150 & $3.62 \mathrm{E}-05$ & -1.11138 & Phospholipase D family, member 5 \\
\hline NEGR 1 & 257194 & $1.97 \mathrm{E}-05$ & -1.10062 & Neuronal growth regulator 1 \\
\hline GRIA2 & 2891 & $1.42 \mathrm{E}-03$ & -1.08966 & Glutamate receptor, ionotropic, AMPA 2 \\
\hline NPR1 & 4881 & $8.88 \mathrm{E}-05$ & -1.07677 & Natriuretic peptide receptor A/guanylate cyclase A \\
\hline ACTC1 & 70 & $2.93 \mathrm{E}-04$ & -1.07237 & Actin, $\alpha$, cardiac muscle 1 \\
\hline PDZRN3 & 23024 & $2.55 \mathrm{E}-05$ & -1.07182 & PDZ domain-containing ring finger 3 \\
\hline LPHN3 & 23284 & $3.67 \mathrm{E}-04$ & -1.06682 & Latrophilin 3 \\
\hline PLN & 5350 & $9.84 \mathrm{E}-05$ & -1.06111 & Phospholamban \\
\hline NLGN1 & 22871 & $6.43 \mathrm{E}-05$ & -1.03802 & Neuroligin 1 \\
\hline THRB & 7068 & $1.16 \mathrm{E}-04$ & -1.0368 & Thyroid hormone receptor, $\beta$ \\
\hline TTLL7 & 79739 & $7.12 \mathrm{E}-05$ & -1.02607 & Tubulin tyrosine ligase-like family, member 7 \\
\hline GRIA1 & 2890 & $3.08 \mathrm{E}-04$ & -1.02562 & Glutamate receptor, ionotropic, AMPA 1 \\
\hline FRK & 2444 & $4.00 \mathrm{E}-04$ & -1.01427 & Fyn-related kinase \\
\hline FREM1 & 158326 & $1.57 \mathrm{E}-04$ & -1.00531 & FRAS1 related extracellular matrix 1 \\
\hline ACP5 & 54 & $4.63 \mathrm{E}-04$ & 1.00142 & Acid phosphatase 5 , tartrate resistant \\
\hline PRDM1 & 639 & $3.75 \mathrm{E}-05$ & 1.005106 & PR domain containing 1 , with $\mathrm{ZNF}$ domain \\
\hline CR1 & 1378 & $7.47 \mathrm{E}-04$ & 1.011272 & Complement component $(3 \mathrm{~b} / 4 \mathrm{~b})$ receptor 1 (Knops blood group) \\
\hline VAV3 & 10451 & $3.72 \mathrm{E}-05$ & 1.026726 & Vav 3 guanine nucleotide exchange factor \\
\hline CXCL10 & 3627 & $5.23 \mathrm{E}-04$ & 1.051734 & Chemokine (C-X-C motif) ligand 10 \\
\hline NPL & 80896 & $5.61 \mathrm{E}-05$ & 1.066364 & $\mathrm{~N}$-acetylneuraminate-pyruvate lyase \\
\hline ITGAX & 3687 & $1.91 \mathrm{E}-04$ & 1.070598 & Integrin, $\alpha \mathrm{X}$ (complement component 3 receptor 4 subunit) \\
\hline RGS1 & 5996 & $5.42 \mathrm{E}-03$ & 1.094815 & Regulator of G-protein signaling 1 \\
\hline CYTIP & 9595 & $1.22 \mathrm{E}-04$ & 1.099515 & Cytohesin 1 interacting protein \\
\hline PLEK & 5341 & $4.59 \mathrm{E}-05$ & 1.104737 & Pleckstrin \\
\hline CD163 & 9332 & $3.84 \mathrm{E}-05$ & 1.130551 & CD163 molecule \\
\hline ADAMDEC1 & 27299 & $2.95 \mathrm{E}-03$ & 1.138718 & ADAM-like, decysin 1 \\
\hline SELE & 6401 & $2.36 \mathrm{E}-04$ & 1.157189 & Selectin E \\
\hline ANPEP & 290 & $8.68 \mathrm{E}-05$ & 1.173833 & Alanyl (membrane) aminopeptidase \\
\hline CCR1 & 1230 & $3.94 \mathrm{E}-05$ & 1.179454 & Chemokine (C-C motif) receptor 1 \\
\hline PLIN2 & 123 & $1.37 \mathrm{E}-04$ & 1.18298 & Perilipin 2 \\
\hline AQP9 & 366 & $1.82 \mathrm{E}-03$ & 1.252998 & Aquaporin 9 \\
\hline CD52 & 1043 & $1.35 \mathrm{E}-04$ & 1.263187 & CD52 molecule \\
\hline CHI3L1 & 1116 & $4.86 \mathrm{E}-03$ & 1.289639 & Chitinase 3-like 1 (cartilage glycoprotein-39) \\
\hline IL1RN & 3557 & $5.61 \mathrm{E}-05$ & 1.394489 & Interleukin 1 receptor antagonist \\
\hline HMOX1 & 3162 & $4.75 \mathrm{E}-05$ & 1.419502 & Heme oxygenase (decycling) 1 \\
\hline MME & 4311 & $8.26 \mathrm{E}-05$ & 1.485 & Membrane metallo-endopeptidase \\
\hline MMP12 & 4321 & $3.59 \mathrm{E}-03$ & 1.574106 & Matrix metallopeptidase 12 \\
\hline DPP4 & 1803 & $3.69 \mathrm{E}-05$ & 1.610873 & Dipeptidyl-peptidase 4 \\
\hline CD36 & 948 & $1.99 \mathrm{E}-04$ & 1.802205 & CD36 molecule (thrombospondin receptor) \\
\hline MMP9 & 4318 & $1.86 \mathrm{E}-04$ & 1.817804 & Matrix metallopeptidase 9 \\
\hline MMP7 & 4316 & $5.72 \mathrm{E}-04$ & 1.840231 & Matrix metallopeptidase 7 \\
\hline FABP4 & 2167 & $2.39 \mathrm{E}-05$ & 2.454461 & Fatty acid binding protein 4 , adipocyte \\
\hline
\end{tabular}

Adj.P, P-value from hypergeometric test adjusted by multiple test adjustment (Benjamini-Hochberg method); DEGs, differentially expressed genes; miRNA or miR, microRNA. 
Patient samples and RNA isolation. Serum specimens were collected from 10 patients with acute ischemia stroke (average age of $69 \pm 10$ ) and 10 healthy individuals (no disease) who received a health check-up. There were no significant differences in age and gender distributions between the patients with acute ischemic stroke and healthy controls $(\mathrm{P}>0.05)$.

The acute ischemic stroke patients were associated with significant infract lesions. Serum samples prior to miRNA analysis were stored at $-80^{\circ} \mathrm{C}$ within $4 \mathrm{~h}$ following collection. The present study adhered to the tenets of the Declaration of Helsinki and was approved by the Medical Ethics Committee of Sichuan University (Sichuan, China). Informed consent was obtained from participants or their close relatives for the use of their blood in this study.

$R T-q P C R$. Total RNA was extracted from serum samples according to a method described previously (31). Synthetic C. elegans miRNA (cel-miR-39; Qiagen, Hilden, Germany) was spiked in each extracted RNA at a final concentration of $4 \mathrm{fmol}$ as an internal control. The concentration and quality of RNA were measured by UV absorbance at 260 and $280 \mathrm{~nm}$ (A260/280 ratio).

Ten nanograms of total RNA was reverse transcribed and qPCR was performed using an All-in-One ${ }^{\mathrm{TM}}$ miRNA qRT-PCR Detection kit (GeneCopoeia, Rockville, MD, USA) according to the manufacturer's instructions. The validated All-in-One miRNA forward primers were purchased from GeneCopoeia. Forward primers specific for cel-miR-39 were designed based on a sequence of mature miRNA obtained from the miRbase (32). The levels of miRNAs were normalized with cel-miR-39. The $2^{-\triangle \Delta C T}$ method, described by Livak et al (33), was used to analyze data. miR-9 in each serum sample and no-template controls were run in triplicate.

Statistical analysis. Statistical analysis was performed using the two-tailed paired Student's t-test using IBM SPSS 21.0 (SPSS, Inc., Chicago, IL, USA). Differences were considered statistically significant at $\mathrm{P}<0.05$.

Confirmed miRNA-targeted gene interaction network. PPIs of DEGs were constructed from the STRING databases (34), and interactions associated with confirmed miRNAs were retained. miRNA-targeted DEGs interaction networks were visualized using the Cytoscape software version 3.1.1 (35).

Hierarchical clustering of expressional values of DEGs. Hierarchical clustering of expression data of the signature DEGs and the confirmed miRNA-targeted DEGs that were altered in atheromatous plaque was performed in a dChip Analyzer (36). The clustering algorithms with Euclidean distance (37) metric and centroid linkage rule were used (36).

GO function and pathway analysis of confirmed miRNAtargeted DEGs interaction. The Cytoscape software with plug-in Bingo was run to determine which biological processes are statistically overrepresented in the set of genes corresponding to the predicted miRNA-targeted DEGs interaction networks (38). The hypergeometric test was employed with a BH-FDR-based multiple testing correction (corr $\mathrm{P}<0.01)$.

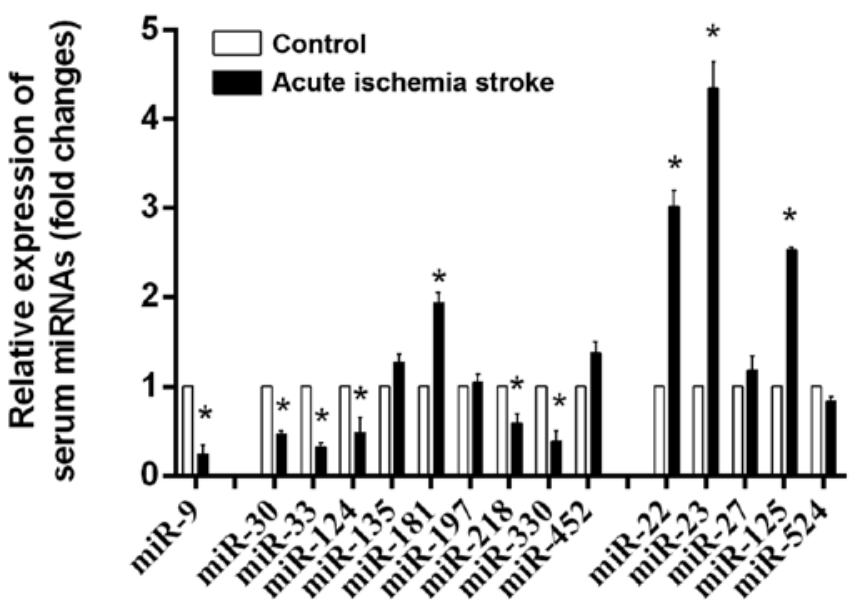

Figure 1. The expression of identified serum microRNAs (miRNAs or miRs) in acute ischemic stroke. Compared to the healthy controls, the expression of serum miRNAs in patients with acute ischemic stroke was tested using reverse transcription quantitative PCR (RT-qPCR). ${ }^{*} \mathrm{P}<0.05$.

\section{Results}

Screening of the DEGs. The expression value was derived for each probe, and an accurate annotation was made. DEGs were identified using the Multtest and Limma packages of $\mathrm{R}$ language. In the present study, 56 and 69 genes that were significantly differentially down- and upregulated, respectively, were identified (data not shown).

Prediction of enriched miRNAs. The top 10 miRNAs significantly associated with the screened up- and downregulated DEGs were predicted using the WebGestalt toolkit (Table I). Six miRNAs were connected with the upregulated DEGs including miR-9, $-22,-23,-27,-125$ and -524 , and 10 miRNAs were connected with the downregulated DEGs including miR-9, -30, -33, -124, -135, -181, -197, -218, -330 and -452. A list of target DEGs associated with these miRNAs is provided in Table I. In the DEGs provided in Table II, the expression of vav 3 guanine nucleotide exchange factor (VAV3) and PR domain containing 1 (PRDM1) was 2.0 -fold higher than that in the intact tissue, and the expression of $\beta$-thyroid hormone receptor (THRB) and contactin 4 (CNTN4) in atheromatous plaque was a notable 2.1- and 3.5-fold lower than that in the intact tissue, respectively. Notably, miR-9 was associated with both down- and upregulated DEGs.

Validation of predicted serum miRNA in acute ischemic stroke. The expression of predicted serum miRNAs in acute ischemic stroke was validated by RT-qPCR (Fig. 1). It was confirmed that the expression of serum miR- $9,-30,-33,-124$, $-181,-218,-330,-22,-23$ and -125 was significantly altered in ischemic stroke, indicating these miRNAs are important in acute ischemic stroke.

Confirmed miRNA-targeted DEGs interaction networks and important DEG nodes. PPIs of DGEs were constructed from the STRING databases. Interactions associated with confirmed miRNAs were retained, and confirmed miRNA-targeted DEGs interaction networks were subsequently visualized 

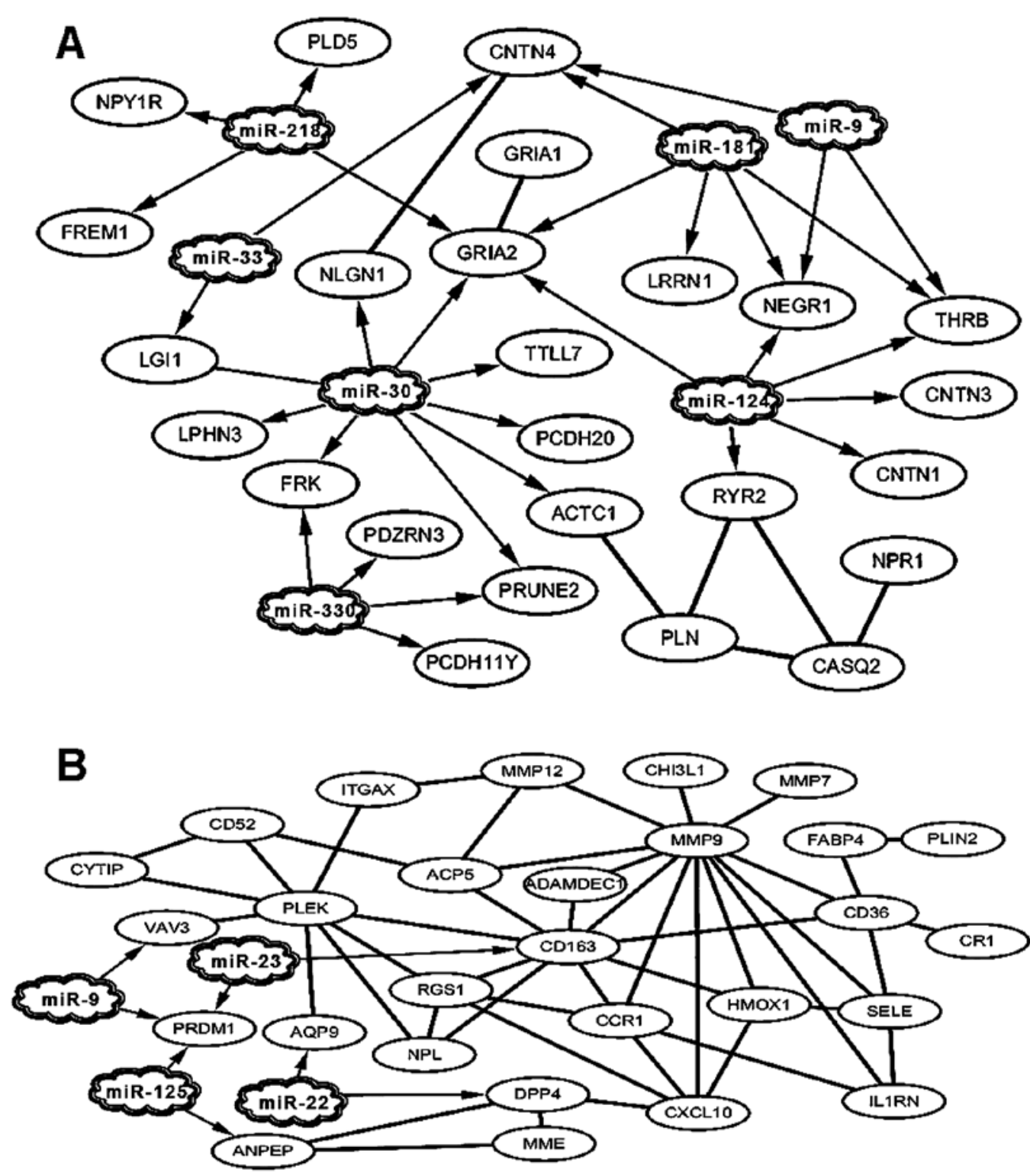

Figure 2. Confirmed microRNA (miRNA or miR)-targeted differentially expressed gene (DEG) interaction networks. Protein-protein interactions (PPIs) of (A) downregulated and (B) upregulated DEGs regulated by miRNAs. The nodes of miRNAs were emphasized in different shapes. Arrows show the associated DEGs.

using Cytoscape software (Fig. 2). Four miRNAs were linked to 25 downregulated DEGs (Fig. 2A) and 7 miRNAs were linked to 28 upregulated DEGs (Fig. 2B) forming miRNAs-DEGs interaction networks. Table II shows the DEGs in the interaction networks. The most important nodes in the miRNA interaction networks were downregulated DEGs including THRB, CNTN4, NEGR1 and glutamate receptor 2 (GRIA2) (Fig. 1A), and upregulated DEGs including VAV3, PRDM1, CD163, matrix metallopeptidase 9 (MMP9), pleckstrin (PLEK), CD36, chemokine (C-X-C motif) ligand 10 (CXCL10), chemokine (C-C motif) receptor 1 (CCR1), regulator of G-protein signaling 1 (RGS1), HMOX1, tartrate resistant acid phosphatase 5 (ACP5), dipeptidyl peptidase 4 (DPP4) and selectin E (SELE) (Fig. 2B).

Hierarchical clustering of expressional values of DEGs. Using the algorithm based on Euclidean distance metric and centroid linkage rule, the hierarchical clustering of expres- sional values of DEGs and the confirmed miRNA-targeted DEGs were divided into the atheromatous plaque and intact tissue groups (Fig. 3). Fig. 3 shows atheromatous plaque and intact tissue samples separated with the exception of 13 paired samples that were not well classified. There were 10 intact tissues clustered in the atheromatous plaque group, and three atheromatous plaques in the intact tissue group, indicating that the differences in gene expression between intact tissues and atheromatous plaques from those patients are extremely close and difficult to distinguish, and that there was a high risk of atheromatous in the 10 patients. Nevertheless, two clustering maps showed identical resampling, suggesting that the confirmed miRNA-targeted DEGs are important representative genes in the interaction networks, and that screening is extremely significant.

GO function and pathway analysis of confirmed miRNAtargeted DEG interaction. GO function suggested that the 


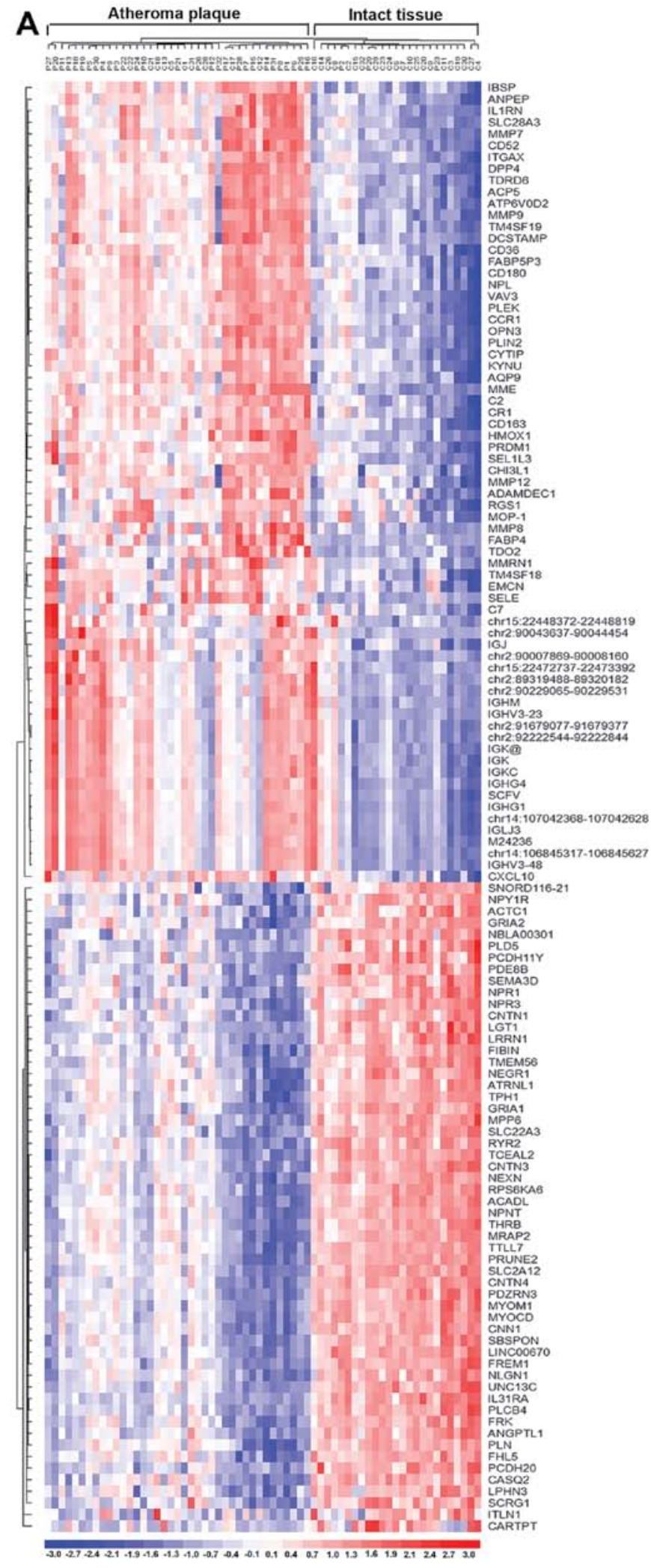

confirmed miRNAs targeting downregulated DEGs were predominately associated with signal transduction, the circulatory system, biological adhesion and striated muscle contraction, and that the targeted upregulated DEGs were most significantly associated with signal transduction, wound healing and the immune system (Table III).
B
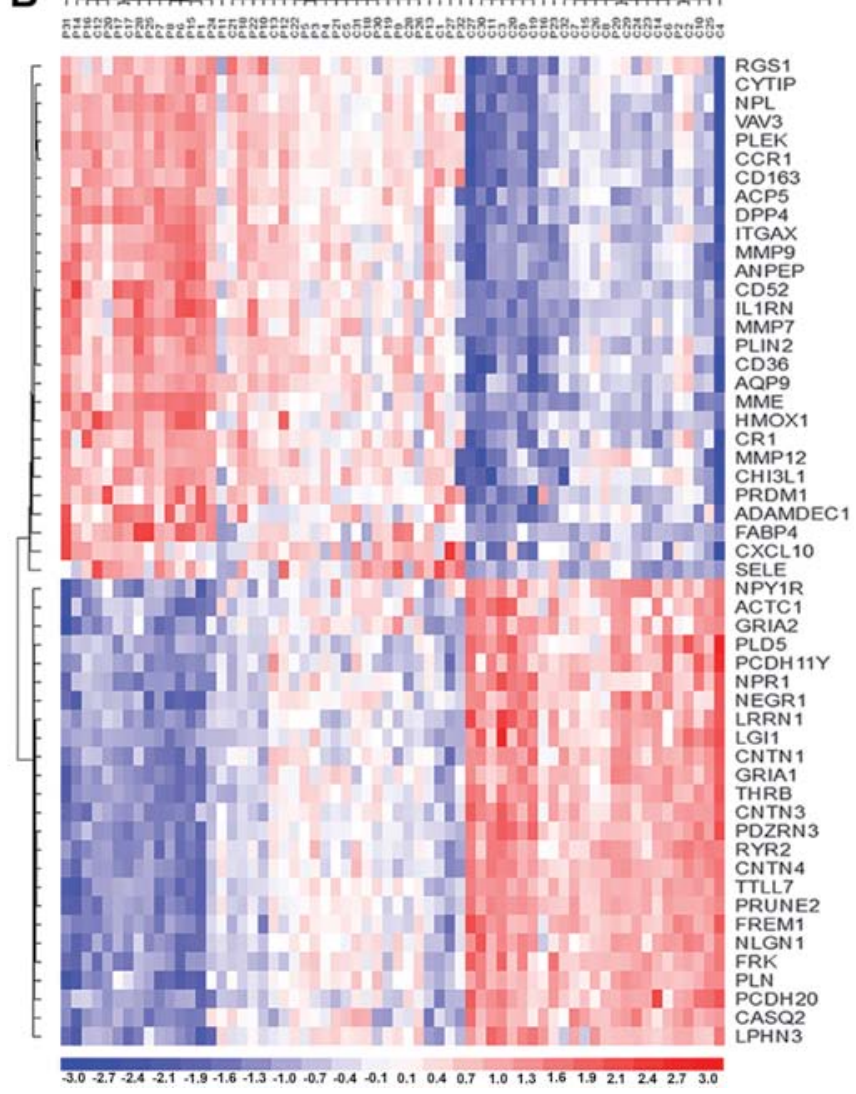

Figure 3. Hierarchical clustering of gene expression profiles. Groups of atheromatous plaque $(\mathrm{P} 1-\mathrm{P} 32)$ and intact tissues $(\mathrm{C} 1-\mathrm{C} 32)$ are represented by $(\mathrm{A})$ the vertical rows and signatures of differentially expressed genes (DEGs), and (B) the microRNA (miRNA)-target DEGs are represented horizontally. On the bottom of the clustering image is the color scale, showing the standardized expression values, since the expression levels for each gene are standardized to have a mean 0 and standard deviation 1 . Red, the expression level above the mean expression of a gene across all the samples. White, the mean expression. Blue, expression lower than the mean. The top hierarchical break distinguished a group of atheromatous plaque from the group of intact tissues.

\section{Discussion}

The predominant etiology of stroke is atherosclerosis (atheroma) (39). The present study provides a list of potential clinical diagnoses and prognostic biomarkers and therapeutic targets for acute ischemic stroke via the identification of a list of DEGs associated with miRNA targets, the validation of expression of predicted miRNAs in acute ischemic stroke, and the GO function analysis of the confirmed miRNAs targeted DEGs in the advanced plaque using the microarray data from Ayari and Bricca (23). Furthermore, a new molecular mechanism causing atherosclerotic plaque rupture and intraplaque hemorrhage, and subsequently ischemic stroke remains to be determined.

Whole-transcript expression profile of human atherosclerotic arteries (endarterectomy specimens) was performed by Ayari and Bricca (23). As it is impossible to obtain normal artery tissue, the intra-patient comparison on the expression profile of atheromatous plaque and intact tissues from the individual was carried out to examine the atherogenic process per se (23). Ayari and Bricca analyzed the expression of two genes (CD163 and HMOX1) involved in the homeostasis of 
Table III. GO enrichment analysis of confirmed miRNAs and targeted DEGs interaction networks.

Downregulated DEGs

\begin{tabular}{lll}
\hline GO-ID & \multicolumn{1}{c}{ Description } & \multicolumn{1}{c}{ Genes in test set } \\
\hline 44057 & Regulation of system process & PLNINLGN1IRYR2INPR1ICNTN4INPY1RILGI1 \\
8015 & Blood circulation & ACTC1IPLNIRYR2INPR1INPY1R \\
3013 & Circulatory system process & ACTC1IPLNIRYR2INPR1INPY1R \\
7155 & Cell adhesion & PCDH11YIFREM1IPCDH20|NLGN1ICNTN1ICNTN4ICNTN3INEGR1 \\
22610 & Biological adhesion & PCDH11YIFREM1IPCDH20|NLGN1ICNTN1ICNTN4ICNTN3INEGR1 \\
6941 & Striated muscle contraction & ACTC1IRYR2ICASQ2 \\
\hline
\end{tabular}

Upregulated DEGs

GO-ID Description Genes in test set

\begin{tabular}{|c|c|c|}
\hline 9611 & Response to wounding & $\begin{array}{l}\text { CR1ICD36|PLEKIHMOX1ICCR1IIL1RNIMMEISELEIMMP12ICD163| } \\
\text { CXCL10 }\end{array}$ \\
\hline 2376 & Immune system process & $\begin{array}{l}\text { CR1 RGS1 PLEK AQP9 CCR1 MMP9 IL1RN ACP5 SELE DPP4 } \\
\text { CXCL10 }\end{array}$ \\
\hline 6950 & Response to stress & $\begin{array}{l}\text { CR1 CD36 PLEK AQP9 HMOX1 CCR1 IL1RN MME ACP5 SELE } \\
\text { MMP12 DPP4 CD163 CXCL10 }\end{array}$ \\
\hline 6954 & Inflammatory response & CR1 HMOX1 CCR1 IL1RN SELE CD163 CXCL10 \\
\hline 7229 & Integrin-mediated signaling pathway & VAV3 PLEK ITGAX ADAMDEC1 \\
\hline 48583 & Regulation of response to stimulus & CR1 PLEK HMOX1 FABP4 ACP5 SELE DPP4 CXCL10 \\
\hline 42221 & Response to chemical stimulus & $\begin{array}{l}\text { PLIN2 AQP9 HMOX1 CCR1 IL1RN FABP4 MME ACP5 } \\
\text { SELE MMP12 DPP4 CXCL10 }\end{array}$ \\
\hline 30155 & Regulation of cell adhesion & CD36 VAV3 CYTIP ADAMDEC1 DPP4 \\
\hline 6952 & Defense response & CR1 HMOX1 CCR1 IL1RN ACP5 SELE CD163 CXCL10 \\
\hline 32101 & Regulation of response to external stimulus & PLEK FABP4 ACP5 SELE CXCL10 \\
\hline 42060 & Wound healing & CD36 PLEK HMOX1 MME MMP12 \\
\hline 60054 & $\begin{array}{l}\text { Positive regulation of epithelial cell } \\
\text { proliferation involved in wound healing }\end{array}$ & MME MMP12 \\
\hline 2685 & Regulation of leukocyte migration & HMOX1 SELE CXCL10 \\
\hline 34383 & Low-density lipoprotein particle clearance & CD36 HMOX1 \\
\hline 50896 & Response to stimulus & $\begin{array}{l}\text { CR1 AQP9 PLEK CCR1 IL1RN ACP5 MME MMP12 CD163 CXCL10 } \\
\text { CD36 RGS1 PLIN2 HMOX1 FABP4 SELE DPP4 }\end{array}$ \\
\hline 15718 & Monocarboxylic acid transport & CD36 PLIN2 AQP9 \\
\hline 48518 & Positive regulation of biological process & $\begin{array}{l}\text { CR1 CD36 VAV3 PLEK HMOX1 MMP9 FABP4 MME PRDM1 SELE } \\
\text { MMP12 DPP4 CXCL10 }\end{array}$ \\
\hline 42493 & Response to drug & PLIN2 AQP9 FABP4 MME MMP12 \\
\hline 6955 & Immune response & CR1 RGS1 AQP9 CCR1 IL1RN ACP5 CXCL10 \\
\hline 10033 & Response to organic substance & PLIN2 AQP9 HMOX1 IL1RN FABP4 ACP5 SELE CXCL10 \\
\hline 8284 & Positive regulation of cell proliferation & HMOX1 FABP4 MME MMP12 DPP4 CXCL10 \\
\hline
\end{tabular}

GO, gene ontology; DEG, differentially expressed genes; miRNA, microRNA. corr $\mathrm{P}<0.01$.

iron and heme, and their role in atheromatous plaques (23). In the present study, we identified 56 downregulated DEGs and 69 upregulated DEGs (including CD163 and HMOX1). A 2 -fold change with FDR $<0.05$ was set as the threshold criterion. Using these DEGs, we screened the associated Top 10 miRNA. Six miRNAs were linked to the upregulated DEGs including miR-9, -22, -23, -27, -125 and -524 , and 10 miRNAs were linked to the downregulated DEGs including miR-9, $-30,-33$,
$-124,-135,-181,-197,-218,-330$ and -452 (Table I). Of these miRNAs, miR-33 and miR-125 were found to associate with hyperlipidemia (19). Notably, miR-9 is an important miRNA associated with down- and upregulated DEGs. The significant change in expression of predicted serum miRNAs (miR-9, -30 , $-33,-124,-181,-218,-330,-22,-23$ and -125$)$ in acute ischemic stroke was validated by RT-qPCR. The results confirmed that miRNAs may be important in acute ischemic stroke. 
PPIs of DGEs were constructed from the STRING databases, and any interactions that were not associated with any miRNA targets were discarded, such as the interaction between calponin 1 (CNN1) and myocardin (MYOCD), which played crucial roles in cardiogenesis and differentiation of the smooth muscle cell lineage (40), as well as the interaction between tryptophan 2,3-dioxygenase (TDO2) and kynureninase (KYNU), which were involved in tryptophan metabolism (41). Interactions associated with confirmed miRNAs were retained, and the confirmed miRNA-targeted DEGs interaction networks were constructed. In total, 25 downregulated DEGs and 28 upregulated DEGs were involved in the miRNAs-DEGs interaction networks.

In the present study, the intact tissues were type I and II lesions, and the atheromatous plaque was type IV lesion and later lesion according to the definition and classification suggested by the Nomenclature Committee of the American Heart Association (24). Of note, type I and II lesions may be combined under the term early lesions, and type IV-VI lesions combined under the term advanced lesions. The early lesions generally are those that occur in infants and children, although they also occur in adults (24). Following the early lesions onset, advanced lesions may subsequently evolve following thickening of the arterial wall and therefore narrowing of the lumen or obstruction or modification of the blood flow. Thus, common gene expression in early and advanced lesions from some individuals may be identical. This hypothesis was supported by the hierarchical clustering maps which did not separate all the endarterectomy specimens into intact tissue and atheromatous plaque groups. Nevertheless, two clustering maps showed identical resampling, indicating that the miRNA-target DEGs were important representative genes in the interaction networks, and that screening is essential.

In the confirmed miRNAs-targeted DEGs interaction networks, the most important nodes identified were THRB, CNTN4, NEGR1 and GRIA2 in the downregulated network, and VAV3, PRDM1, CD163, MMP9, PLEK, CD36, CXCL10, CCR1, RGS1, HMOX1, ACP5, DPP4 and SELE in the upregulated network. Of note, the miR-9 target DEGs including THRB, CNTN4, NEGR1, VAV3 and PRDM1 were important nodes in the miRNAs-target DEGs interaction networks, suggesting that miR-9 plays a central role in the atheroma. The important nodes were enriched in the GO term.

Ischemic stroke occurs when an artery is obstructed (19), which is most often caused by atheroma. Atheroma (atherosclerosis), which is also known as the type VI lesion, is characterized by larger, confluent and more disruptive core of extracellular lipid (24). Almost $70 \%$ of plaque specimens (carotid endarterectomy specimens) demonstrated fissures, hematoma or hemorrhage, and/or thrombus, 64\% demonstrated neovascularization $(14,24)$. Arteries with thinned or ruptured fibrous caps, intraplaque hemorrhage, larger lipid-rich necrotic cores and larger wall thickness were associated with the occurrence of subsequent clinical events, especially the stroke $(19,42)$. In addition, recurrent stroke is associated with a higher incidence of large-artery atherosclerosis than the first stroke (19). The exact mechanisms causing plaque are not yet completely known (15). The plaques that are rich in soft extracellular lipid (43), neoformed vessels and inflammatory infiltration (44) are vulnerable. The fibrous cap at the site of the rupture/erosion had an eroded surface characterized by loss of the endothelial lining (45). As demonstrated in GO annotations: CD36 was associated with lipid storage (GO: 19915); and HOMOX1, CCR1, SELE, CD163 and CXCL10 were associated with the inflammatory response (GO: 6950). miR-9 targeted downregulated DEGs including CNTN4, NEGR1 and THRB were predominately associated with biological adhesion and regulation of the neurological system, and targeted upregulated DEGs including VAV3 and PRDM1 were most significantly associated with signal transduction and vasculature development, showing a crucial role of miR-9 in the vulnerability of plaque. By contrast, the miRNA expression profile in young stroke patients (18-49 years) have not shown a difference in miR-9 (22). Our results supported that miR-9 is a potential biomarker for acute ischemic stroke. We also observed significant changes in miR-30, -33, -124, -181, -218, -330, -22, -23 and -125 expression. However, further investigation focusing on their role in acute ischemic stroke should be conducted. Furthermore, investigation into the molecular mechanism underlying the formation and rupture of atherosclerotic plaque may provide a new therapeutic strategy for the prevention, diagnosis, treatment and prognosis of ischemic strokes.

\section{Acknowledgements}

This study was supported by the National Natural Science Foundation of China (grant no. 11402153), and the Talent Introduction Scientific Research Projects Funded Start-Up Funds of Sichuan University of China (no. 2082204174089).

\section{References}

1. Lopez AD, Mathers CD, Ezzati M, Jamison DT and Murray CJ: Global and regional burden of disease and risk factors, 2001: Systematic analysis of population health data. Lancet 367: 1747-1757, 2006.

2. World Health Organization: The Global Burden of Disease: 2004 Update, 2008.

3. Ding Z, Tong WC, Lu XX and Peng HP: Hyperbaric oxygen therapy in acute ischemic stroke: a review. Interv Neurol 2: 201-211, 2014.

4. Krafft PR, Bailey EL, Lekic T, Rolland WB, Altay O, Tang J, Wardlaw JM, Zhang JH and Sudlow CL: Etiology of stroke and choice of models. Int J Stroke 7: 398-406, 2012.

5. Warlow C, Sudlow C, Dennis M, Wardlaw J and Sandercock P: Stroke. Lancet 362: 1211-1224, 2003.

6. Herson PS, Palmateer J and Hurn PD: Biological sex and mechanisms of ischemic brain injury. Transl Stroke Res 4: 413-419, 2013.

7. Go AS, Mozaffarian D, Roger VL, Benjamin EJ, Berry JD, Blaha MJ, Dai S, Ford ES, Fox CS, Franco S, et al; American Heart Association Statistics Committee and Stroke Statistics Subcommittee: Heart disease and stroke statistics - 2014 update: a report from the American Heart Association. Circulation 129: e28-e292, 2014.

8. Yong H, Foody J, Linong J, Dong Z, Wang Y, Ma L, Meng HJ, Shiff $\mathrm{S}$ and Dayi H: A systematic literature review of risk factors for stroke in China. Cardiology Rev 21: 77-93, 2013.

9. He J, Klag MJ, Wu Z and Whelton PK: Stroke in the People's Republic of China. II. Meta-analysis of hypertension and risk of stroke. Stroke 26: 2228-2232, 1995.

10. Janardhan V and Qureshi AI: Mechanisms of ischemic brain injury. Curr Cardiol Rep 6: 117-123, 2004.

11. Kikuchi K, Miura N, Kawahara KI, Murai Y, Morioka M, Lapchak PA and Tanaka E: Edaravone (Radicut), a free radical scavenger, is a potentially useful addition to thrombolytic therapy in patients with acute ischemic stroke. Biomed Rep 1: 7-12, 2013.

12. Golledge J, Greenhalgh RM and Davies AH: The symptomatic carotid plaque. Stroke 31: 774-781, 2000.

13. Milei J, Parodi JC, Alonso GF, Barone A, Grana D and Matturri L: Carotid rupture and intraplaque hemorrhage: immunophenotype and role of cells involved. Am Heart J 136: 1096-1105, 1998. 
14. Milei J, Parodi JC, Ferreira M, Barrone A, Grana DR and Matturri L: Atherosclerotic plaque rupture and intraplaque hemorrhage do not correlate with symptoms in carotid artery stenosis. J Vasc Surg 38: 1241-1247, 2003.

15. van der Wal AC, Becker AE, van der Loos CM and Das PK: Site of intimal rupture or erosion of thrombosed coronary atherosclerotic plaques is characterized by an inflammatory process irrespective of the dominant plaque morphology. Circulation 89: 36-44, 1994

16. Russo $\mathrm{G}$ and Giordano A: miRNAs: from biogenesis to networks. Methods Mol Biol 563: 303-352, 2009.

17. Chen X, Ba Y, Ma L, Cai X, Yin Y, Wang K, Guo J, Zhang Y, Chen J, Guo X, et al: Characterization of microRNAs in serum: a novel class of biomarkers for diagnosis of cancer and other diseases. Cell Res 18: 997-1006, 2008.

18. Lorenzen JM, Volkmann I, Fiedler J, Schmidt M, Scheffner I, Haller H, Gwinner W and Thum T: Urinary miR-210 as a mediator of acute T-cell mediated rejection in renal allograft recipients. Am J Tranplant 11: 2221-2227, 2011.

19. Rink $C$ and Khanna S: MicroRNA in ischemic stroke etiology and pathology. Physiol Genomics 43: 521-528, 2011.

20. Tan JR, Koo YX, Kaur P, Liu F, Armugam A, Wong PT and Jeyaseelan K: microRNAs in stroke pathogenesis. Curr Mol Med 11: 76-92, 2011.

21. Bates DJ, Li N, Liang R, Sarojini H, An J, Masternak MM, Bartke A and Wang E: MicroRNA regulation in Ames dwarf mouse liver may contribute to delayed aging. Aging Cell 9: 1-18 2010.

22. Tan KS, Armugam A, Sepramaniam S, Lim KY, Setyowati KD, Wang CW and Jeyaseelan K: Expression profile of MicroRNAs in young stroke patients. PLoS One 4: e7689, 2009.

23. Ayari $\mathrm{H}$ and Bricca G: Identification of two genes potentially associated in iron-heme homeostasis in human carotid plaque using microarray analysis. J Biosci 38: 311-315, 2013.

24. Stary HC, Chandler AB, Dinsmore RE, Fuster V, Glagov S, Insull W Jr, Rosenfeld ME, Schwartz CJ, Wagner WD and Wissler RW: A definition of advanced types of atherosclerotic lesions and a histological classification of atherosclerosis. A report from the Committee on Vascular Lesions of the Council on Arteriosclerosis, American Heart Association. Circulation 92 1355-1374, 1995.

25. Fujita A, Sato JR, Rodrigues LO, Ferreira CE and Sogayar MC: Evaluating different methods of microarray data normalization. BMC Bioinformatics 7: 469, 2006

26. Butte AJ, Ye J, Häring HU, Stumvoll M, White MF and Kohane IS: Determining significant fold differences in gene expression analysis. Pac Symp Biocomput 2001: 6-17, 2001.

27. Gentleman R, Carey V, Huber W, Irizarry RA and Dudoit S (eds): Bioinformatics and computational biology solutions using $\mathrm{R}$ and Bioconductor. Vol. 746718470. Springer-Verlag, New York, NY, 2005.

28. Benjamini $\mathrm{Y}$ and Hochberg Y: Controlling the false discovery rate: a practical and powerful approach to multiple testing. J R Stat Soc 57: 289-300, 1995.

29. Zhang B, Kirov S and Snoddy J: WebGestalt: An integrated system for exploring gene sets in various biological contexts. Nucleic Acids Res 33 (Suppl 2): W741-W748, 2005.
30. Duncan D, Prodduturi N and Zhang B: WebGestalt2: An updated and expanded version of the Web-based Gene Set Analysis Toolkit. BMC Bioinformatics 11 (Suppl 4): 10, 2010.

31. Lodes MJ, Caraballo M, Suciu D, Munro S, Kumar A and Anderson B: Detection of cancer with serum miRNAs on an oligonucleotide microarray. PLoS One 4: e6229, 2009.

32. Griffiths-Jones S, Saini HK, van Dongen S and Enright AJ: miRBase: tools for microRNA genomics. Nucleic Acids Res 36: D154-D158, 2008.

33. Livak KJ and Schmittgen TD: Analysis of relative gene expression data using real-time quantitative PCR and the 2(-Delta Delta C(T)) Method. Methods 25: 402-408, 2001.

34. Szklarczyk D, Franceschini A, Kuhn M, Simonovic M, Roth A, Minguez P, Doerks T, Stark M, Muller J, Bork P, et al: The STRING database in 2011: Functional interaction networks of proteins, globally integrated and scored. Nucleic Acids Res 39 (Suppl 1): D561-D568, 2011.

35. Smoot ME, Ono K, Ruscheinski J, Wang P-L and Ideker T: Cytoscape 2.8: new features for data integration and network visualization. Bioinformatics 27: 431-432, 2011.

36. Li C and Hung Wong W: Model-based analysis of oligonucleotide arrays: model validation, design issues and standard error application. Genome Biol 2: RESEARCH0032, 2001.

37. Deza MM and Deza E: Encyclopedia of distances. Springer, Berlin 2009.

38. Maere S, Heymans K and Kuiper M: BiNGO: a Cytoscape plugin to assess overrepresentation of gene ontology categories in biological networks. Bioinformatics 21: 3448-3449, 2005.

39. Kim JS and Bonovich D: Research on intracranial atherosclerosis from the East and west: why are the results different? J Stroke 16: 105-113, 2014.

40. Lv B, Zhao J, Yang F, Huang X, Chen G, Yang K, Liu S, Fan C, $\mathrm{Fu} \mathrm{H}$ and Chen Z: Phenotypic transition of corpus cavernosum smooth muscle cells subjected to hypoxia. Cell Tissue Res 357: 823-833, 2014

41. Miller CL, Murakami P, Ruczinski I, Ross RG, Sinkus M, Sullivan B and Leonard S: Two complex genotypes relevant to the kynurenine pathway and melanotropin function show association with schizophrenia and bipolar disorder. Schizophr Res 113: 259-267, 2009.

42. Takaya N, Yuan C, Chu B, Saam T, Underhill H, Cai J, Tran N, Polissar NL, Isaac C, Ferguson MS, et al: Association between carotid plaque characteristics and subsequent ischemic cerebrovascular events: a prospective assessment with MRI - initial results. Stroke 37: 818-823, 2006.

43. Falk E: Why do plaques rupture? Circulation 86 (Suppl): III30-III42, 1992.

44. Ribatti D, Levi-Schaffer F and Kovanen PT: Inflammatory angiogenesis in atherogenesis - a double-edged sword. Ann Med 40: 606-621, 2008

45. Milei J, Parodi JC, Fernandez Alonso G, Barone A, Beigelman R, Ferreira LM, Arrigoni G and Matturri L: Carotid atherosclerosis. Immunocytochemical analysis of the vascular and cellular composition in endarterectomies. Cardiologia 41: 535-542, 1996. 